\title{
Treg depletion potentiates checkpoint inhibition in claudin-low breast cancer
}

\author{
Nicholas A. Taylor, ${ }^{1}$ Sarah C. Vick, ${ }^{1,2}$ Michael D. Iglesia, ${ }^{3}$ W. June Brickey, ${ }^{1,2}$ Bentley R. Midkiff, ${ }^{1}$ Karen P. Mckinnon, ${ }^{1,2}$ \\ Shannon Reisdorf, ${ }^{1}$ Carey K. Anders, ${ }^{1,4}$ Lisa A. Carey, ${ }^{1,4}$ Joel S. Parker, ${ }^{1,3}$ Charles M. Perou, ${ }^{1,3}$ \\ Benjamin C. Vincent, ${ }^{1,4,5}$ and Jonathan S. Serody ${ }^{1,2,4}$
}

'Lineberger Comprehensive Cancer Center, ${ }^{2}$ Department of Microbiology and Immunology, ${ }^{3}$ Department of Cenetics, ${ }^{4}$ Department of Medicine, and ${ }^{5} \mathrm{Curriculum}$ in Bioinformatics and Computational Biology,

University of North Carolina (UNC), Chapel Hill, North Carolina, USA

\begin{abstract}
Claudin-low breast cancer is an aggressive subtype that confers poor prognosis and is found largely within the clinical triplenegative group of breast cancer patients. Here, we have shown that intrinsic and immune cell gene signatures distinguish the claudin-low subtype clinically as well as in mouse models of other breast cancer subtypes. Despite adaptive immune cell infiltration in claudin-low tumors, treatment with immune checkpoint inhibitory antibodies against cytotoxic T lymphocyteassociated protein 4 (CTLA-4) and programmed death receptor 1 (PD-1) were ineffective in controlling tumor growth. $\mathrm{CD}^{+}{ }^{+} \mathrm{FoxP3}^{+}$Tregs represented a large proportion of the tumor-infiltrating lymphocytes (TILs) in claudin-low tumors, and Tregs isolated from tumor-bearing mice were able to suppress effector T cell responses. Tregs in the tumor microenvironment highly expressed PD-1 and were recruited partly through tumor generation of the chemokine CXCL12. Antitumor efficacy required stringent Treg depletion combined with checkpoint inhibition; delays in tumor growth were not observed using therapies that modestly diminished the number of Tregs in the tumor microenvironment. This study provides evidence that the recruitment of Tregs to the tumor microenvironment inhibits an effective antitumor immune response and highlights early Treg recruitment as a possible mechanism for the lack of response to immune checkpoint blockade antibodies in specific subtypes of cancer that are heavily infiltrated with adaptive immune cells.
\end{abstract}

\section{Introduction}

Breast cancer is the leading cancer diagnosis and second-leading cause of cancer death for women in the United States (1). Patient prognosis is determined largely by stratification based on age, lymph node involvement, tumor grade, and tumor cell-surface expression of estrogen receptor (ER), progesterone receptor (PR), and human epidermal growth factor receptor 2 (HER2) (2-7). Early diagnosis and targeted therapies, both hormonal and immunologic, are critical for the effective treatment of ER-, PR-, and HER2-positive disease. Given the lack of targeted therapies for patients whose tumors do not express ER, $\mathrm{PR}$, and HER2, termed triple-negative breast cancers, the survival benefit from recent therapeutic advances has eluded this group of patients. This is especially important for clinicians and patients, as triple-negative tumors present with higher grade at diagnosis and an increased incidence for disease relapse, early metastasis, and death (8-11).

Transcriptome profiling has defined 5 intrinsic molecular subtypes of breast cancer: luminal A, luminal B, HER2 enriched, basal-like, and claudin-low $(12,13)$. The basal-like and claudin-low

Authorship note: N.A. Taylor and S.C. Vick are co-first authors. B.G. Vincent and J.S Serody are co-senior authors.

Conflict of interest: The authors have declared that no conflict of interest exists.

Submitted: September 6, 2016; Accepted: July 11, 2017

Reference information: J Clin Invest. 2017;127(9):3472-3483.

https://doi.org/10.1172/JCI90499. subtypes are distinguished by increased expression of genes associated with tumor cell proliferation and adaptive and innate immunity $(14,15)$. Claudin-low tumors are further separated from basal-like tumors by their decreased expression of cell-cell adhesion claudin proteins, increased expression of genes associated with epidermal-to-mesenchymal transition, and stem cell-like characteristics (16). Importantly, basal-like and claudin-low tumors make up the majority of triple-negative tumors and have a significantly poorer prognosis than luminal and HER2-enriched tumors (17).

In addition to tumor-intrinsic genetic features, tumor growth is dependent on tumor-extrinsic factors, which include the tumor stroma and the local immune response $(14,18)$. Tumor-infiltrating lymphocytes (TILs)can have divergent effects in the tumor microenvironment (TME). Whereas the presence of multiple immune cell types may herald productive antitumor immunity (15), TILs may also contribute to tumor progression through immune suppression or the production of soluble factors that regulate cell proliferation, migration, and angiogenesis (19).

While the presence of TILs is associated with an improvement in the survival of patients with multiple different tumor subtypes, this has not been found for specific subtypes of breast cancer. The luminal A subtype, which exhibits no appreciable immune infiltration, carries the best prognosis, whereas the basal-like and claudin-low subtypes exhibit a marked immune infiltrate with worse clinical outcomes (20). In this study, we sought to understand the mechanism by which immune infiltration is associated with poor clinical outcomes for patients with claudin-low tumors. 
A

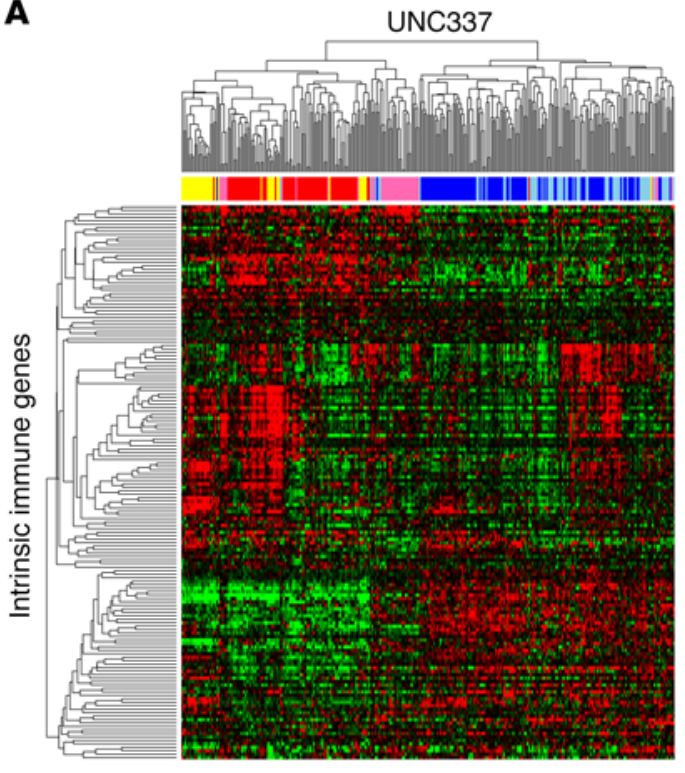

Tumor subtypes:

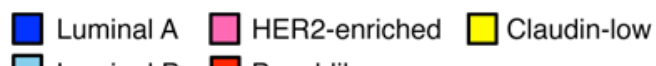

$\square$ Luminal B $\square$ Basal-like

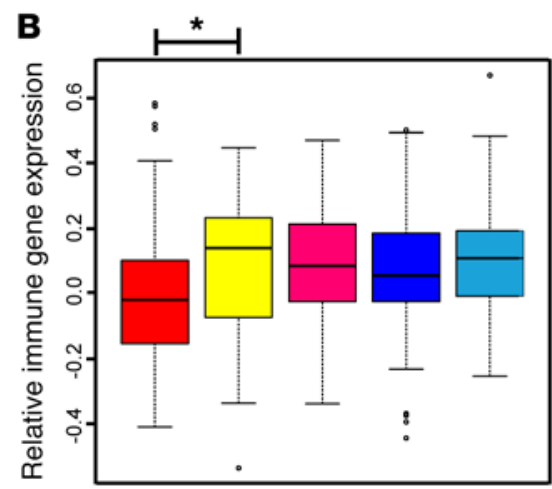

Tumor subtype

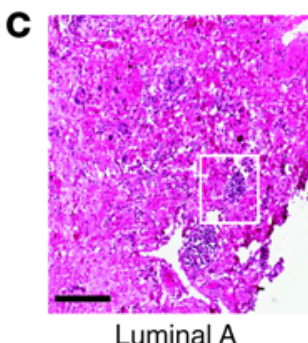

Luminal A

E

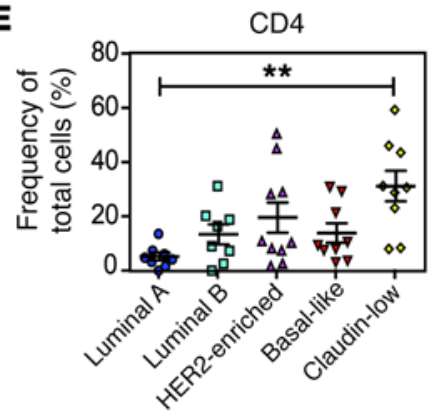

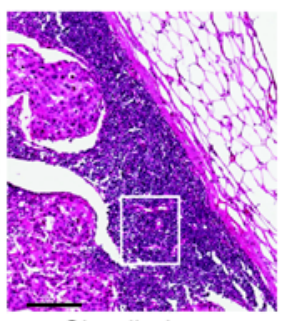

Claudin-low

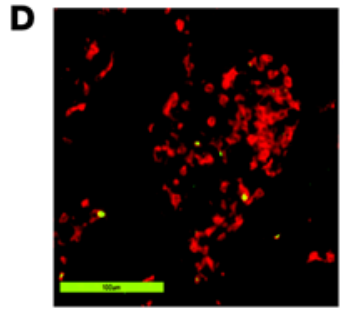

Luminal A

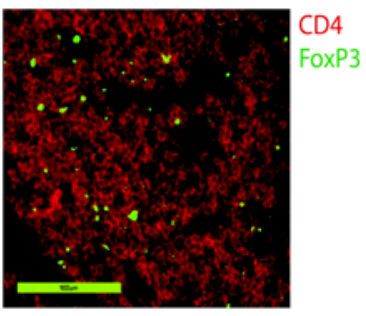

Claudin-low

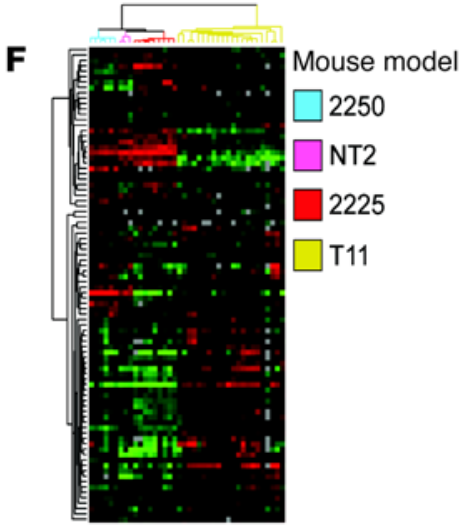

Figure 1. Human tumors exhibit differential immune gene expression. (A) Unsupervised hierarchical clustering of breast cancer samples from UNC337 by IIG list. (B) Overall expression of the immune gene signature by each subtype. (C) Representative H\&E staining of human subtype-specific breast cancer. Original magnification, $\times 5$. (D) Representative IF staining of human tumors for CD4 (red) and FoxP3 (green). Original magnification, $\times 20$. (E) Whole-slide scoring of CD4+ infiltrate for each subtype. (F) Unsupervised clustering by the IIGs in GEMMs $(n=59)$. Statistical significance determined by Kruskal-Wallis test, with Dunn's post-test for multiple comparisons. Data are presented as mean \pm SEM. ${ }^{*} P<0.05 ;{ }^{* *} P<0.01$.

We show that patients with claudin-low tumors have an increased number of $\mathrm{CD}^{+}{ }^{+} \mathrm{T}$ cells with focal increases in Tregs in the TME compared with other intrinsic subtypes. To understand the mechanism for this, the $\mathrm{p} 53^{---} \mathrm{T} 11$ and $\mathrm{T} 12$ genetically engineered mouse models (GEMMs) were used, which closely recapitulated the cell-intrinsic and -extrinsic characteristics of human claudin-low breast cancer. In human and mouse studies, gene expression profiling revealed increased expression of immune genes and Treg-associated genes in the claudin-low subtype. Importantly, checkpoint inhibition was found to be effective for claudin-low tumors only when combined with stringent Treg depletion. Taken together, these experiments highlight the role of Tregs in suppressing the immune response in claudin-low breast tumors and provide an understanding of the differential response to immune checkpoint inhibition in breast cancer.

\section{Results}

Claudin-low tumors show increased expression of immune genes relative to other intrinsic subtypes of breast cancer. The original microarray used to classify the intrinsic subtypes of breast cancer included over 1,700 genes $(21,22)$, with an emphasis on cell-intrinsic transcriptional characteristics. We were interested in determining whether there were cell-extrinsic transcriptional differences that could be used to classify breast cancer subtypes. For this analysis, we evaluated the expression of 102 immune genes that encompass downstream effector molecules, chemokines and chemokine receptors, immune response signal mediators, cell-specific markers, and markers of oxidative stress, termed the intrinsic immune genes (IIGs) (Supplemental Table 1; supplemental material available online with this article; https://doi.org/10.1172/ 
JCI90499DS1). Gene expression profiling by microarray analysis was done on 337 breast tumors as previously described (23). Unsupervised hierarchical clustering of UNC337 by only those genes in the IIG list divided patients into 3 clusters: low-expressing luminal, moderate-expressing HER2 enriched, and high-expressing basal-like and claudin-low tumors (Figure 1A). By gene expression signature quantification, HER2-expressing tumors had significantly lower immune gene expression than claudin-low tumors $(P<0.05)$ (Figure 1B). Furthermore, claudin-low tumors showed significantly increased expression of a gene signature associated with Treg activity $(P=0.001$, data not shown).

To confirm these findings, we performed transcriptome evaluation using the IIG classifier from a previous published breast cancer database (Molecular Taxonomy of Breast Cancer International Consortium [METABRIC]; http://www.cbioportal.org/ study?id=brca_metabric\#summary), as the stringency filter for tumor purity eliminated the inclusion of claudin-low tumors from the TCGA database (https://cancergenome.nih.gov/). Unsupervised hierarchical clustering of the METABRIC data by only those genes in the IIG list revealed that clinical breast cancer samples divided into 3 clusters: low-expressing luminal, moderateexpressing HER2 enriched, and high-expressing basal-like and claudin-low tumors similar to what was shown in the UNC337 data (Supplemental Figure 1). The claudin-low subtype had a significantly increased immune gene expression compared with the other subtypes in the METABRIC data set $(P<0.0001)$.

Claudin-low breast tumors show increased infiltration by $\mathrm{CD}^{+}$ cells. In order to determine whether IIG expression was associated with differences in Th cells, Tregs, or cytotoxic T cells (Tc cells), patient biopsy samples were studied by histology and immunofluorescence (IF). Figure 1C shows H\&E stains of the greatest area of immune infiltration of a representative luminal A and claudin-low tumor. Tumor tissue slides were analyzed for Tc cells $\left(\mathrm{CD} 8^{+}\right)$, Th cells $\left(\mathrm{CD}^{+}{ }^{+} \mathrm{FoxP}^{-}\right)$, and Tregs $\left(\mathrm{CD}^{+}{ }^{+} \mathrm{FoxP}^{+}\right)$. Representative images of CD4 and FoxP3 IF staining from the highlighted areas shown in Figure $1 \mathrm{C}$ demonstrate $\mathrm{CD}^{+} \mathrm{FoxP}^{+}$cell infiltration into the tumor (Figure 1D). Claudin-low tumors recruited significantly more $\mathrm{CD} 4^{+} \mathrm{T}$ cells to the tumor site than luminal A tumors $(P=0.002$; Figure $1 \mathrm{E})$, with areas of focally increased numbers of Tregs (Figure 1D). No differences in the number of CD8 ${ }^{+}$ $\mathrm{T}$ cells were found across the different intrinsic subtypes (data not shown). These data indicate the heterogeneity of the immune response in breast cancer subtypes, with claudin-low tumors having a significantly increased $\mathrm{T}$ cell infiltrate $(P<0.01)$ with focally increased numbers of Tregs. In contrast, luminal tumors showed an extremely limited number of $\mathrm{CD}^{+}$and/or $\mathrm{CD} 4^{+} \mathrm{T}$ cells.

Claudin-low GEMMs closely recapitulate human claudin-low breast cancer. Given the difficulties with standardizing tumor latency using clinical samples and the extreme difficulty evaluating the TME at the onset of tumor formation, we sought an in vivo method to better evaluate mechanisms for immune infiltration in claudin-low breast cancer. For this analysis, we used GEMMs of the claudin-low subtype, which have been found to be genotypically similar to human claudin-low tumors (24). Unsupervised clustering of the GEM models using the IIG list (Figure 1F) recreated the clustering profile seen in human samples with very modest expression of immune genes in 2250 tumors (luminal A) compared with very high expression in T11 tumors (claudin-low). We generated a cell line from T11 tumors to standardize claudin-low tumor growth in vivo. Gene expression profiling of these tumors showed that the parental T11 tumor and tumors derived from injection of the T11 cell line clustered tightly together within the claudin-low subtype, demonstrating that the T11 cell line is genetically quite similar to T11 tumor cells (Supplemental Figure 2).

Claudin-low tumors recruit increased numbers of immune cells to the tumor. Tumors representing the subtypes luminal A, HER2/Neu, and claudin-low were studied in GEMMs to evaluate the immune response during tumor progression. In order to evaluate both early and late time points, tumors were harvested at approximately 20 $\mathrm{mm}^{2}$ (Figure 2, A-D) or $100 \mathrm{~mm}^{2}$ (Figure 2, E-H), respectively, and analyzed by flow cytometry to determine the composition of TILs over time. FACS analysis work flows are shown in Supplemental Figure 3, and representative FACS plots are shown in Supplemental Figure 4. The total number of immune cells was significantly increased in T11 claudin-low tumors (Figure 2, A-H). Specifically, the number, but not the frequency, of $\mathrm{CD}^{+} \mathrm{FoxP}^{-} \mathrm{T}$ cells was increased in the TME in both $20-\mathrm{mm}^{2}$ and $100-\mathrm{mm}^{2}$ tumors (Figure 2, A and E). Interestingly, we found an increase in the number of Tregs both at $20 \mathrm{~mm}^{2}$ and $100 \mathrm{~mm}^{2}$ in the T11 claudin-low model. There was a statistically significant increase in the number of CD19+ B cells in mice, with $20 \mathrm{~mm}^{2}$ T11 claudin-low compared with Neu tumors, and a significant increase in B cells in mice with $100 \mathrm{~mm}^{2}$ T11 tumors compared with luminal A tumors (Figure 2, C and G). Additionally, we found very few $\mathrm{CD}^{+} \mathrm{T}$ cells in the TME of mice with either luminal A- or Neu-expressing tumors. There was a statistically significant increase in the number and frequency of $\mathrm{CD}^{+}$ $\mathrm{T}$ cells in mice with $20-\mathrm{mm}^{2}$ and $100-\mathrm{mm}^{2}$ claudin-low tumors (Figure 2, B and F). There was an inverse correlation between the presence of Tregs and diminished number of both $\mathrm{CD} 8^{+} \mathrm{T}$ cells and

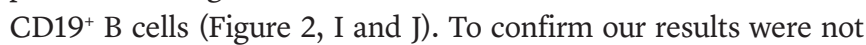
specific to the T11 cell line, we utilized an additional claudin-low tumor cell termed T12 to examine immune infiltration into the TME at late-stage tumor growth. We found an increase in Tregs in mice with $100 \mathrm{~mm}^{2}$ tumors in the $\mathrm{T} 12$ model, although the increase was not significant. We investigated the activation status of the cells migrating into the TME by looking at naive and memory T cell populations (Supplemental Figure 5). Compared with luminal A- and Neu-expressing tumors, the T11 and T12 claudin-low tumors had increased effector memory cells in both the $\mathrm{CD}^{+}{ }^{+} \mathrm{FoxP}^{-}$and $\mathrm{CD}^{+}$ T cell populations. (Supplemental Figure 5, B and D). Thus, these data indicated that there was a significant difference in the population of immune cells in the TME in mouse luminal A, Neu-expressing, or claudin-low tumors. Additionally, our data suggest that the early presence of Tregs was associated with diminished numbers of $\mathrm{CD}^{+} \mathrm{T}$ cells and B cells in mice with claudin-low tumors.

Tregs in mice with tumors show enhanced suppression and chemokine production. Next, we evaluated whether the Tregs isolated from tumor-bearing mice were efficient at suppressing $\mathrm{T}$ cell responses. As shown (Figure 3, A and B), Tregs were capable of suppressing $\mathrm{T}$ cell proliferation in a mixed lymphocyte reaction assay. At high ratios of Treg/T effector cells (Teffs), Tregs were more effective when isolated from mice with $100-\mathrm{mm}^{2}$ compared with $20-\mathrm{mm}^{2}$ tumors. Thus, tumor-bearing mice have functional Tregs that display enhanced suppression in mice with larger tumors. 
A
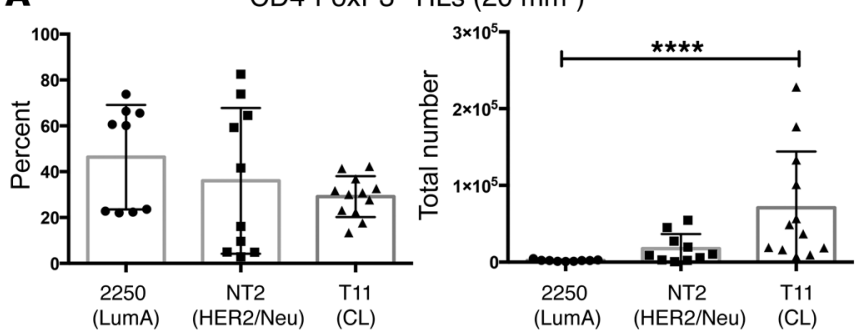

C
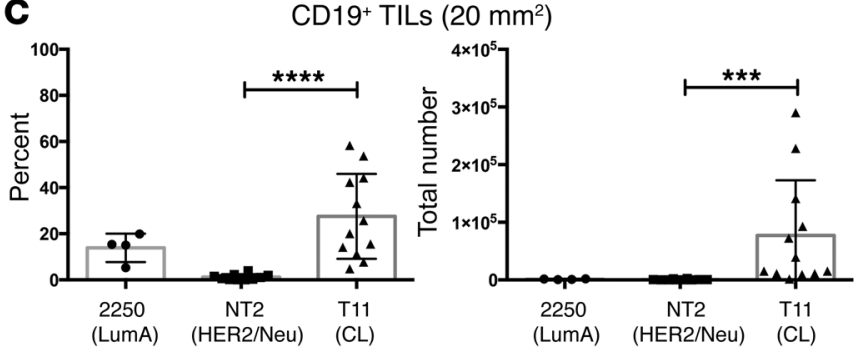

E
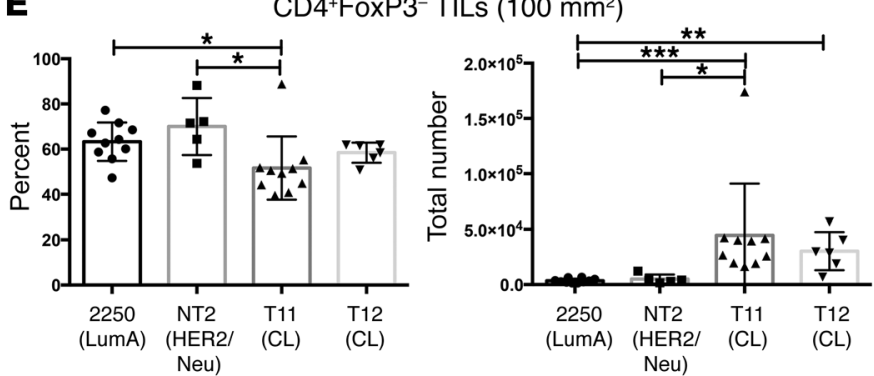

G
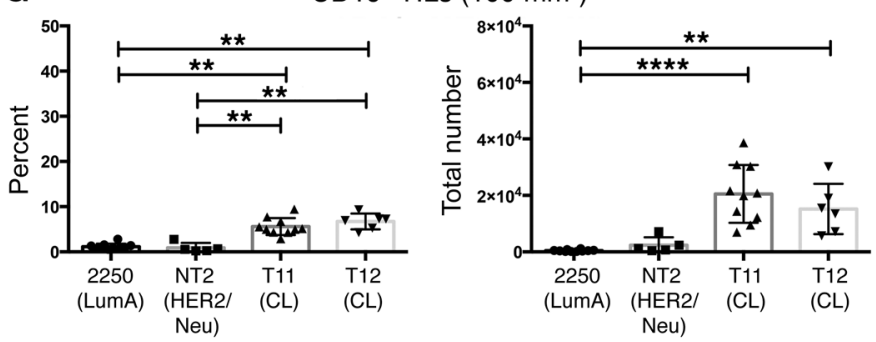

T11 CD8 immune

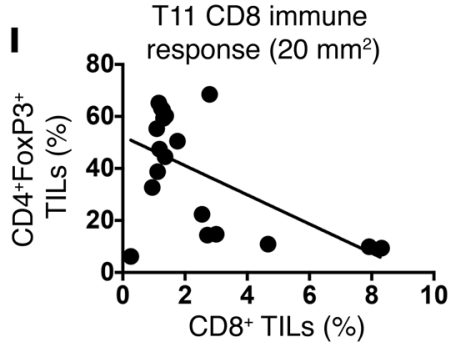

B

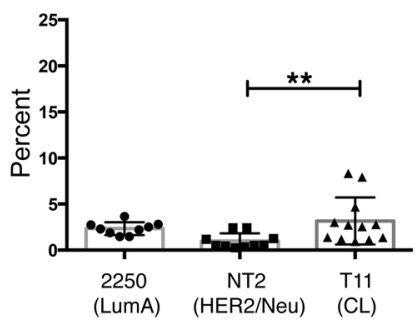

CD8 ${ }^{+}$TILs $\left(20 \mathrm{~mm}^{2}\right)$

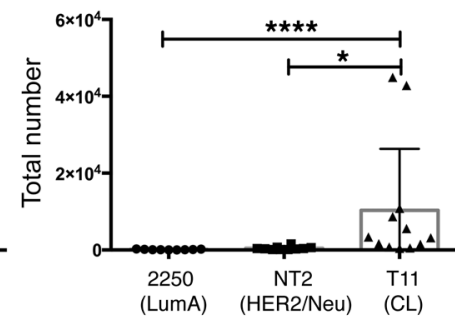

D
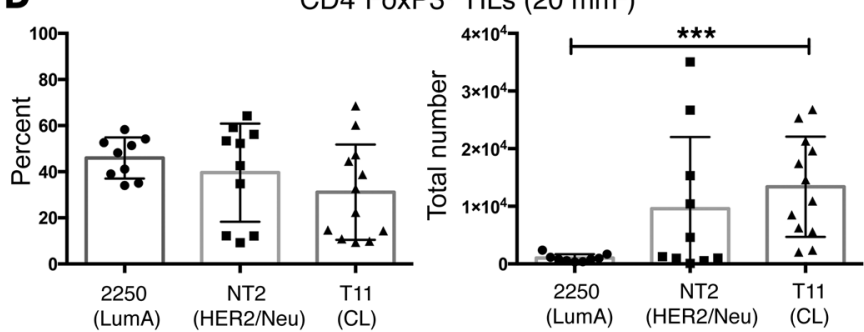

$\mathbf{F}$

CD8 ${ }^{+}$TILs $\left(100 \mathrm{~mm}^{2}\right)$
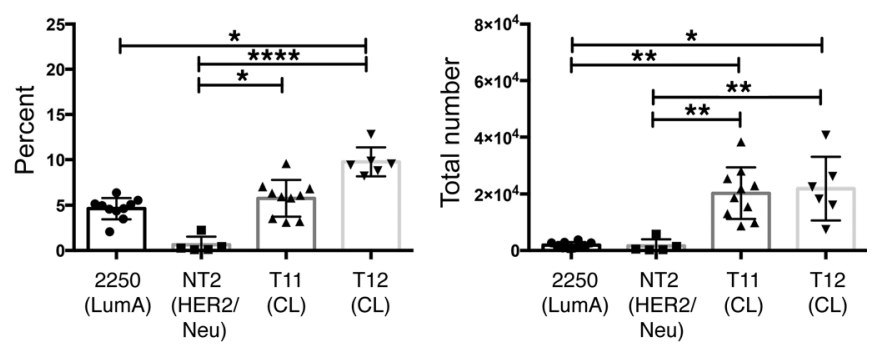

H
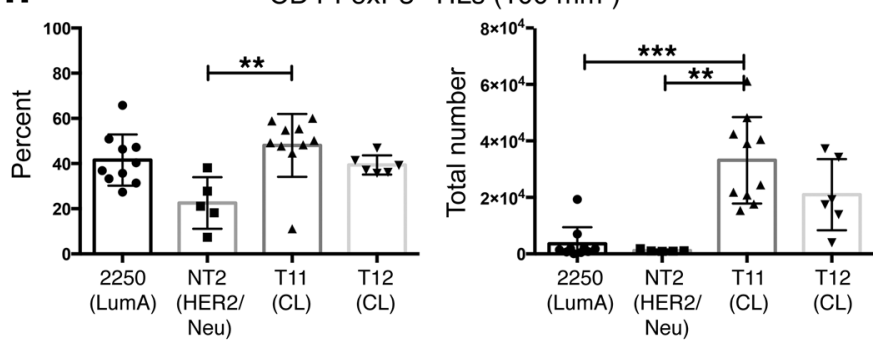

Figure 2. T11 and T12 (claudin-low) tumors recruit elevated numbers of immune cells to the tumor site. WT mice were injected with $1 \times 10^{6} 2250$ tumor cells or $1 \times 10^{4} \mathrm{~T} 11$ or T12 cells. Neu-N mice were injected with $5 \times 10^{4} \mathrm{NT2}$ cells. Tumors were harvested at $20 \mathrm{~mm}^{2}(2250, n=9 ; \mathrm{NT2}, n=10 ; \mathrm{T11}, n=12)(\mathbf{A}-\mathbf{D})$ or $100 \mathrm{~mm}^{2}(2250, n=10 ; \mathrm{NT2}, n=5 ; \mathrm{T} 11, n=10 ; \mathrm{T} 12, n=6)(\mathbf{E}-\mathbf{H})$, digested, enriched for lymphocytes, and analyzed by FACS. (A) CD4 ${ }^{+}$TILs, $20 \mathrm{~mm}{ }^{2}$. (B)

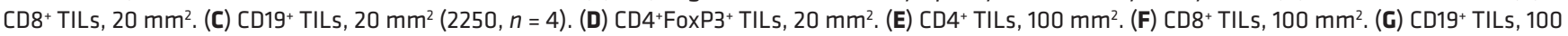
$\mathrm{mm}^{2}$. (H) CD4 ${ }^{+} \mathrm{FoxP3}^{+} \mathrm{TILs}, 100 \mathrm{~mm}^{2}$. (I) Correlation between the percentage of CD8 ${ }^{+} \mathrm{T}$ cells and FoxP3 ${ }^{+} \mathrm{CD} 4^{+} \mathrm{T}$ cells in mice with $20 \mathrm{~mm}^{2} \mathrm{~T} 11$ tumors. $n=16$ mice. (J) Correlation between CD19+ B cells and FoxP3 ${ }^{+} \mathrm{CD}^{+}$T cells in T11 mice with $20 \mathrm{~mm}^{2}$ tumors. $n=16$ (taken from the same group as evaluated in I). Statistical significance determined by Kruskal-Wallis test with Dunn's post-test for multiple comparisons. Data are presented as mean $\pm \mathrm{SEM}$. ${ }^{*} P<0.05$; ${ }^{* *} P<0.01 ;{ }^{* *} P<0.001 ;{ }^{* * *} P<0.0001$. 


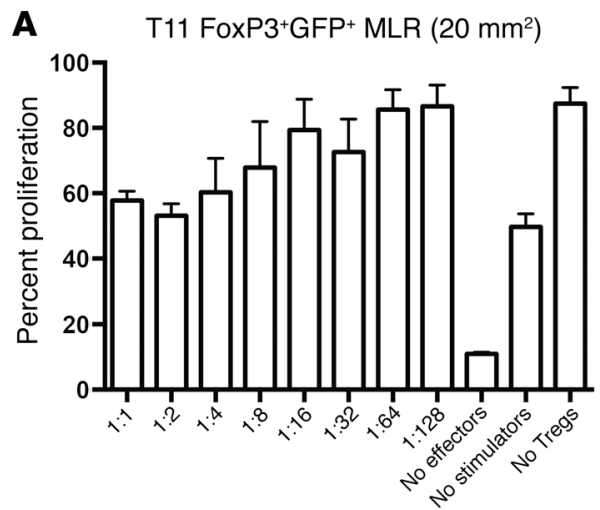

Treg/Teff ratio

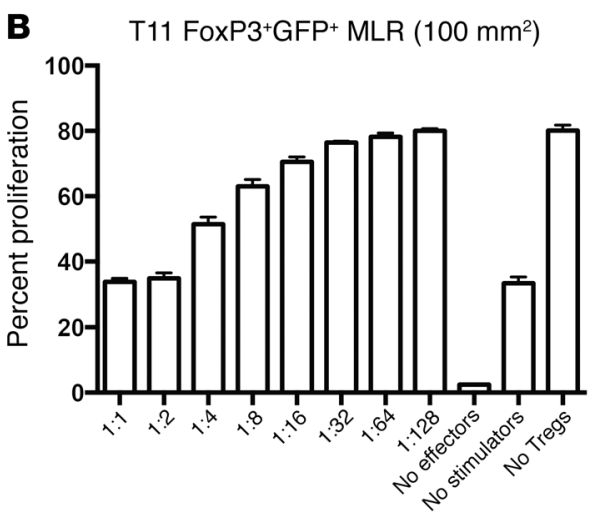

Treg/Teff ratio
Figure 3. FoxP3 cells from T11 tumorbearing mice are functionally suppressive. FoXP3 ${ }^{\text {GFP }}$ mice were injected with 5 $\times 10^{4} \mathrm{~T}^{11}$ cells. GFP+FoxP3 ${ }^{+}$splenocytes were sorted from tumor-bearing mice at (A) $20 \mathrm{~mm}^{2}$ or (B) $100 \mathrm{~mm}^{2}$ and plated with irradiated allogeneic stimulator cells and different ratios of WT Teffs for 3 days. Teffs were then FACS analyzed for dilution of proliferation dye. $n=3$ mice at each tumor size. Data are presented as mean \pm SEM.
To determine what mediators were responsible for recruiting Tregs to the claudin-low tumor site, we compared gene expression differences between Neu-expressing and claudin-low tumors (Figure 4A). T11 tumors expressed significantly higher levels of multiple chemokines, with CXCL12 being 22-fold higher in these tumors compared with Neu-expressing tumors (95\% CI: 17.1-28.3; $P<0.001)$. ELISA of tumor homogenate for CXCL12 confirmed the array findings, with claudin-low tumors expressing higher levels of CXCL12 protein than Neu-overexpressing tumors (Figure 4B). Elevated levels of CXCL12 protein were also found in the serum of claudin-low tumor-bearing mice (Figure 4C). Tregs that infiltrate T11 tumors demonstrated higher expression levels of CXCR4, a receptor for CXCL12, compared with conventional $\mathrm{CD}^{+} \mathrm{T}$ cells $(P=0.03)$ or $\mathrm{CD}^{+} \mathrm{T}$ cells $(P=0.003$; Figure $4, \mathrm{D}$ and $\mathrm{E})$.

To evaluate whether the CXCL12/CXCR4 axis was critical to the recruitment of Tregs in mice with claudin-low tumors, mice were treated with the CXCR4 inhibitor AMD3100. There was a trend toward decreased Treg numbers in AMD3100-treated mice (Figure 5A), which was greater when comparing the number of FoxP3 ${ }^{+} \mathrm{PD}-1^{+}$Tregs, where PD-1 indicates programmed death receptor $1(P=0.07)$ (Figure $5 \mathrm{~B})$. To further evaluate whether the CXCL12/CXCR4 axis was critical to the recruitment of Tregs, shRNA knockdown of CXCL12 was achieved by lentiviral expression of CXCL12-targeted shRNA into T11 tumor cells. (Figure 5, $\mathrm{C}$ and D). We generated 2 cell lines, one with a $55 \%$ knockdown of CXCL12 (KD1) and one with an 85\% knockdown (KD5). There was a nonsignificant reduction in both Tregs and PD $-1^{+}$Tregs in the TME using the more robust knockdown of CXCL12 at day 13 post tumor implantation (PTI) (Figure 5, E and F). Thus, these data indicate that inhibition of the CXCL12/CXCR4 axis in vivo had a modest but not significant effect on Treg accumulation in claudin-low tumors.

Checkpoint inhibition therapy does not alter T11 tumor growth. Because of the increased number of immune cells infiltrating the tumor, we hypothesized that the T11 claudin-low model would be responsive to checkpoint inhibition therapy with blocking antibodies against the inhibitory receptors PD-1 and cytotoxic T lymphocyte-associated protein 4 (CTLA-4). Surprisingly, checkpoint inhibition therapy conferred no benefit on tumor growth or survival in the T11 claudin-low model (Figure 6, E and F), despite expression of PD- 1 on $\mathrm{CD}^{+} \mathrm{T}$ cells (Figure 6A). This result was confirmed in the T12 claudin-low model (Supplemental Figure 6).
However, and unexpectedly, FACS analysis of the immune cells infiltrating the tumor showed that Tregs had the highest levels of PD- 1 and CTLA-4 expression, as measured by FACS mean fluorescence intensity (MFI) (Figure 6, A-D).

Depletion of Tregs delays T11 tumor growth. The inverse correlation between the presence of Tregs and the absence of CD $8^{+} \mathrm{T}$ cells and $\mathrm{CD} 19^{+} \mathrm{B}$ cells in the TME (Figure 2, I and J) led us to hypothesize that approaches that target Tregs would enhance the antitumor immune response. To deplete Tregs, we made use of mice in which the diphtheria toxin (DT) receptor had been knocked in to the FoxP3 locus (DEREG mice) and treated with DT to specifically deplete Tregs in vivo. DEREG mice given DT exhibited a significant delay in tumor growth and a nonsignificant improvement in survival (Figure 7, A and B), with all mice eventually succumbing to either autoimmunity or tumor. As an alternative nongenetic approach to treatment, tumor-bearing WT mice received a single treatment of low-dose $(100 \mathrm{mg} / \mathrm{kg})$ cyclophosphamide (Cy) to selectively deplete Tregs (25). As with DT treatment of DEREG mice, a single injection of low-dose Cy significantly delayed tumor growth (Figure 7, A and B), but again, all mice eventually succumbed to death upon tumor administration. While Treg numbers were decreased compared with those in untreated T11 tumors after Cy, they had increased to above baseline by the time tumors reached $20 \mathrm{~mm}^{2}$ (Figure 7C). Thus, Treg depletion alone appeared to modestly affect tumor growth.

Depletion of Tregs plus checkpoint inhibition improved survival and increased cytokine production by $C D 8^{+} T$ cells. While depleting Tregs delayed tumor growth, we evaluated whether addition of checkpoint inhibition therapy would enhance the benefit. Given the finding that blocking CXCR4 had a modest effect on the recruitment of Tregs in T11 tumors, we initially evaluated whether combining checkpoint inhibition with AMD3100 would have an impact on tumor growth. There were no synergistic effects between CXCR4 inhibition and checkpoint inhibition, as we found no difference in tumor growth using combined checkpoint inhibition and control treatment or AMD3100 (Supplemental Figure 7, A and B). Mice receiving AMD3100 had a reduction in Tregs 12 days after the onset of treatment (Supplemental Figure 7, C and D), but by day 16 , the reduction in infiltrating immune cells was no longer specific to Tregs, as we saw a reduction in $\mathrm{CD} 4^{+}$and $\mathrm{CD} 8^{+} \mathrm{T}$ cells (Supplemental Figure 7, E and F). When combined with checkpoint inhibition, all cell subsets were significantly reduced in the 

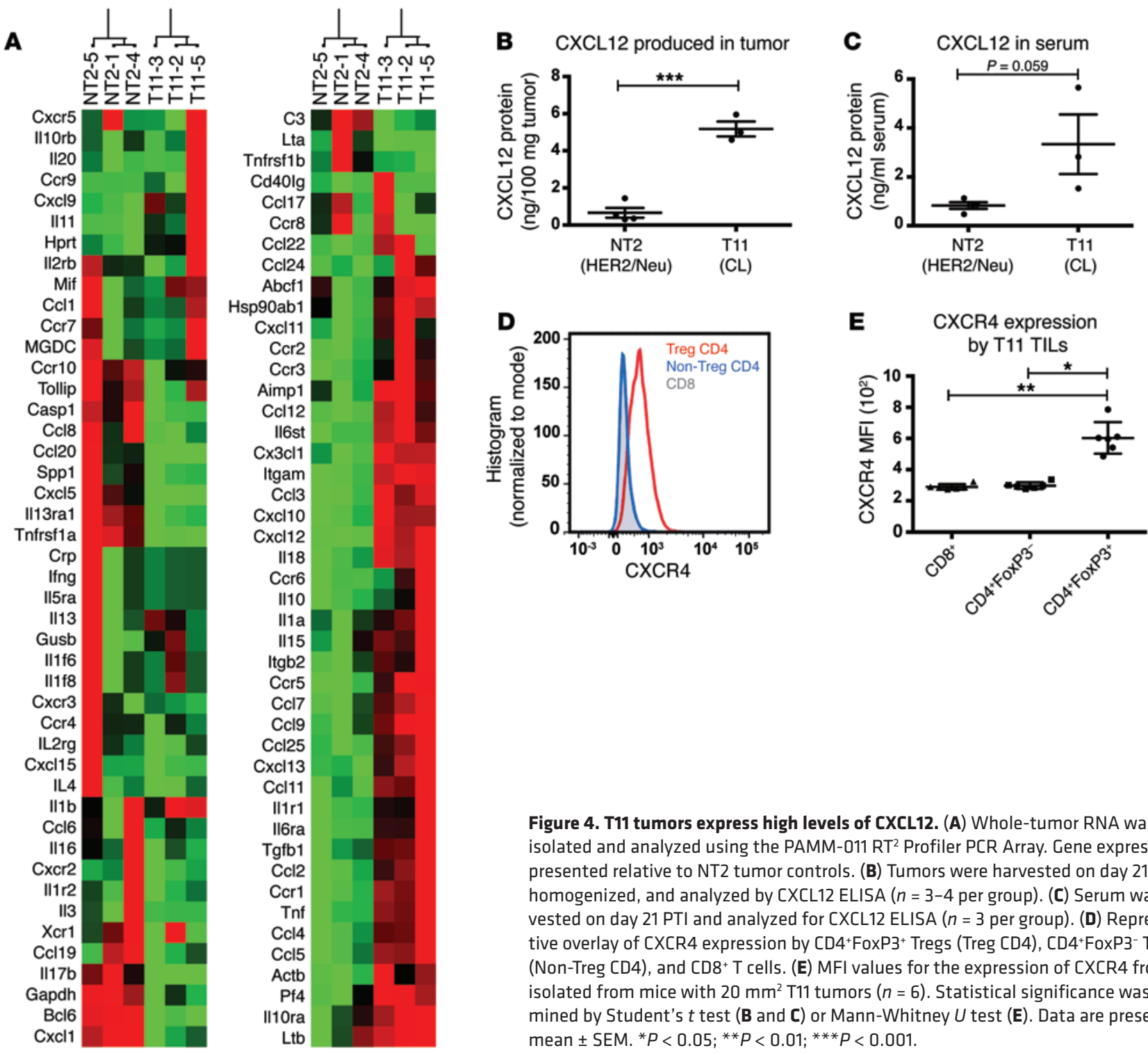

Figure 4. T11 tumors express high levels of CXCL12. (A) Whole-tumor RNA was isolated and analyzed using the PAMM-011 RT ${ }^{2}$ Profiler PCR Array. Gene expression is presented relative to NT2 tumor controls. (B) Tumors were harvested on day 21 PTI, homogenized, and analyzed by CXCL12 ELISA ( $n=3-4$ per group). (C) Serum was harvested on day 21 PTI and analyzed for CXCL12 ELISA ( $n=3$ per group). (D) Representative overlay of CXCR4 expression by $\mathrm{CD}^{+} \mathrm{FoxP3}^{+}$Tregs (Treg CD4), CD4 ${ }^{+} \mathrm{FoxP3}^{-} \mathrm{T}$ cells (Non-Treg CD4), and CD8 ${ }^{+}$T cells. (E) MFI values for the expression of CXCR4 from TILs isolated from mice with $20 \mathrm{~mm}^{2}$ T11 tumors $(n=6)$. Statistical significance was determined by Student's $t$ test (B and $\mathbf{C}$ ) or Mann-Whitney $U$ test (E). Data are presented as mean \pm SEM. ${ }^{*} P<0.05$; ${ }^{* *} P<0.01$; ${ }^{* *} P<0.001$.

TME (Supplemental Figure 7, C-E). As a more rigorous test, Tregs were depleted using DT treatment in DEREG mice in combination with anti-CTLA-4 and anti-PD-1 monoclonal antibody treatment. Tregs were depleted from approximately $20 \%$ to $5 \%$ after DT treatment (Figure 8F). T11 tumor-bearing mice were followed for 25 days PTI until tumor growth reached $170 \mathrm{~mm}^{2}$ or mice developed significant autoimmunity from Treg depletion. Treg depletion in combination with anti-PD-1 and anti-CTLA-4 therapy resulted in decreased tumor growth and significantly improved survival $(P=0.03)$ (Figure 8, A and B). Unfortunately, because mice treated with DT developed severe autoimmunity, we were unable to extend the tumor growth curves past 25 days PTI per institutional IACUC guidelines. We then sought to determine whether delayed tumor growth was correlated with increased cytokine production by $\mathrm{T}$ cells. We analyzed IFN- $\gamma$ production by $\mathrm{CD} 8^{+} \mathrm{T}$ cells in both the tumor and the spleen by FACS as a measure of productive $\mathrm{CD}^{+} \mathrm{T}$ cell responses. While this evaluation was limited greatly by the significant occurrence of autoimmunity in mice treated with DT, there was a nonsignificant increase in the percentage of
$\mathrm{CD}^{+} \mathrm{T}$ cells that generated IFN- $\gamma$, but not in total $\mathrm{CD}^{+} \mathrm{T}$ cells, in mice treated with DT that also received checkpoint inhibition therapy (Figure 8, C-E).

To circumvent the issues with autoimmunity in mice given DT treatment prior to tumor implantation, we treated a small group of mice with DT PTI. While we were limited again in the ability to generate significant numbers of DEREG mice to evaluate, there was prolonged survival until day 40 for half of the mice given DT therapy PTI (Supplemental Figure 8). Because of the autoimmunity associated with the use of DEREG mice, we sought a pharmacological approach to suppress Treg function in vivo. Previous work has shown that selective inhibition of the p110 $\delta$ isoform of PIK3 using a small molecule inhibitor delayed 4T1 tumor growth in mice and reduced Treg suppression (26). We used the small molecule inhibitor PI-3065 in addition to checkpoint inhibition in our T11 claudin-low model. Treatment with PI-3065 was associated with a statistically significant improvement in survival, with a period of tumor stability in half of the treated mice (Supplemental Figure 9, A and B). However, we were not able to mediate tumor 
A

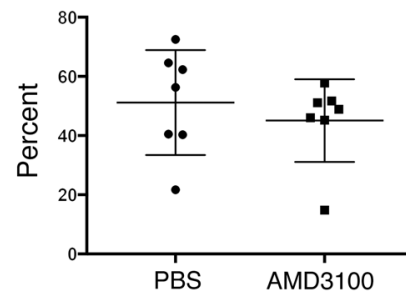

CD4 ${ }^{+} \mathrm{FoxP}^{+}{ }^{+} \mathrm{TILS}$

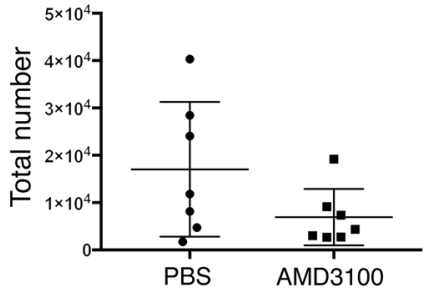

B

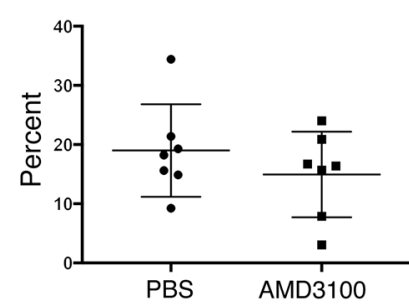

CD4+FoxP3+PD1+ TILs

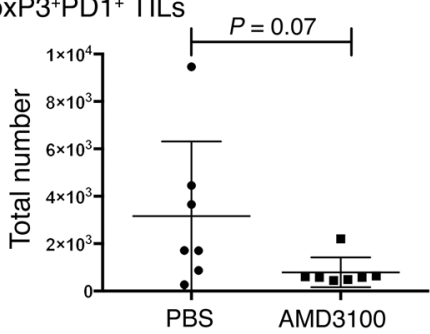

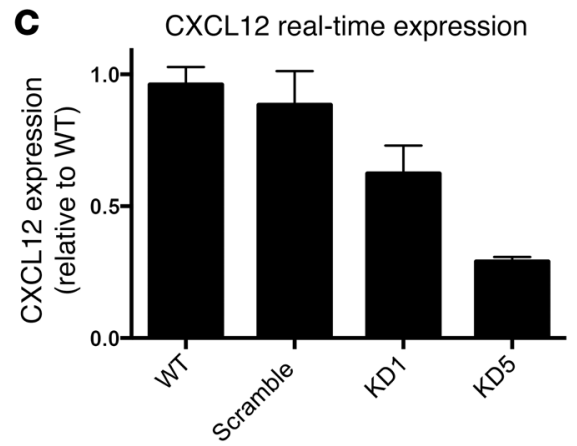

E

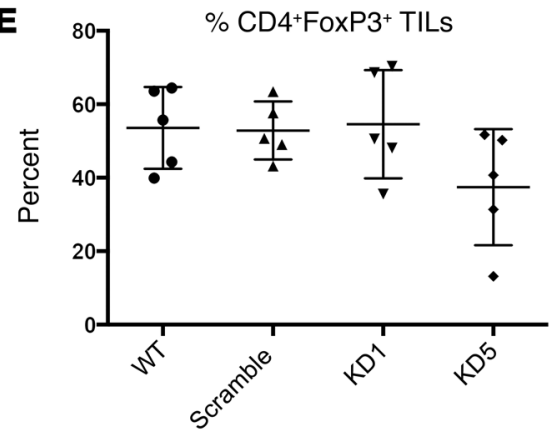

$\mathbf{F}$

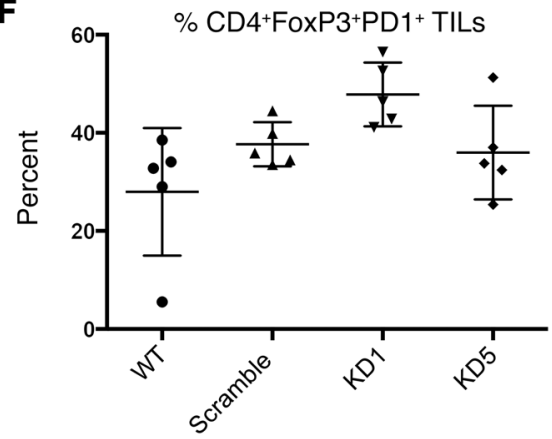

D
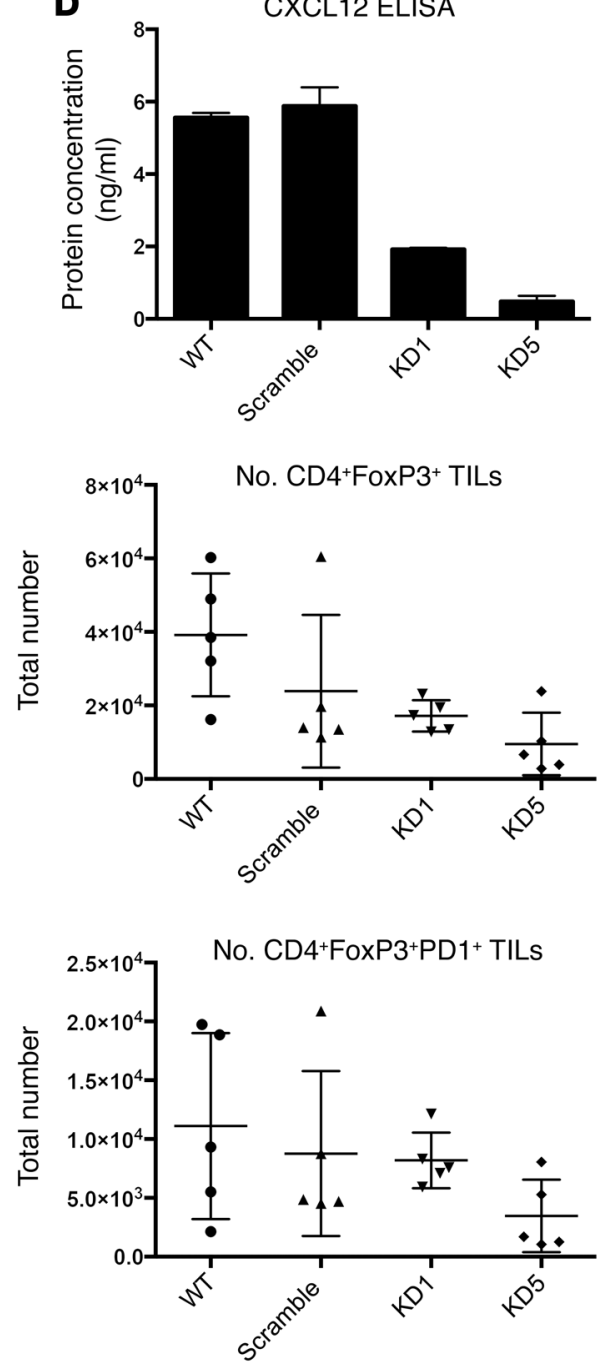

Figure 5. Role of CXCL12/CXCR4 pathway in Treg infiltration into claudin-low tumors. (A and B) WT mice were implanted on day -2 with osmotic pumps loaded with PBS or 10 mg AMD3100 in PBS ( $n=7$ per group) and challenged with $1 \times 10^{4} \mathrm{~T} 11$ cells. Tumors were harvested on day $12 \mathrm{PTI}$ and FACS analyzed. (A) Percentage and number of CD4+FoxP3 ${ }^{+}$TILs. (B) Percentage and number of CD4 ${ }^{+}$FoxP3 ${ }^{+} P D-1^{+}$TILs. (C) T11 cell line was transfected with lentiviral-expressing CXCL12-targeted shRNA, and (D) CXCL12 knockdown was confirmed using CXCL12 ELISA. (E and F) WT mice were injected with $1 \times 10^{4}$ cells of WT, scramble, or knockdown (KD) lines ( $n=5$ per group). Tumors were harvested on day 13 PTI and FACS analyzed. (E) Percentage and number of CD4 ${ }^{+} \mathrm{FoxP3}^{+}$TILs. (F) Percentage and number of $\mathrm{CD} 4^{+} \mathrm{FoxP3} 3^{+} \mathrm{PD}-1^{+}$TILs. Statistical significance was determined by Mann-Whitney $U$ test. Data are presented as mean $\pm \mathrm{SEM}$. regression or affect overall survival. Treatment with PI-3065 and checkpoint therapy did significantly reduce the number of Tregs infiltrating into the tumor (Supplemental Figure 8C). However, it also led to a reduction in the number of $\mathrm{CD}^{+}{ }^{+} \mathrm{FoxP} 3$-negative $\mathrm{T}$ cells accumulating in the TME.

\section{Discussion}

Triple-negative breast cancer has the worst prognosis of the histologic subtypes due to presenting at higher grade at diagnosis, increased recurrence risk following treatment, and shortened time to metastasis (27). The paradoxical observation that the molecular subtypes comprising these tumors also exhibit high lev- els of immune cell infiltration suggests that these infiltrating cells may have a deleterious impact on patient outcome. Our study supports this conclusion for claudin-low breast cancer. Clustering by the IIG alone was sufficient to separate triple-negative basal-like and claudin-low tumors from luminal tumors and HER2-enriched tumors. This finding was not due to aberrant gene expression by tumor cells, but to increased immune cell infiltration into the tumor, especially CD $4^{+} \mathrm{T}$ cells. These results are in contrast with those in prior studies where numbers of TILs were found to be favorably prognostic in breast cancer (5). Importantly, these studies did not stratify breast tumors by molecular subtype, highlighting that the significance of the immune infiltrate may vary 

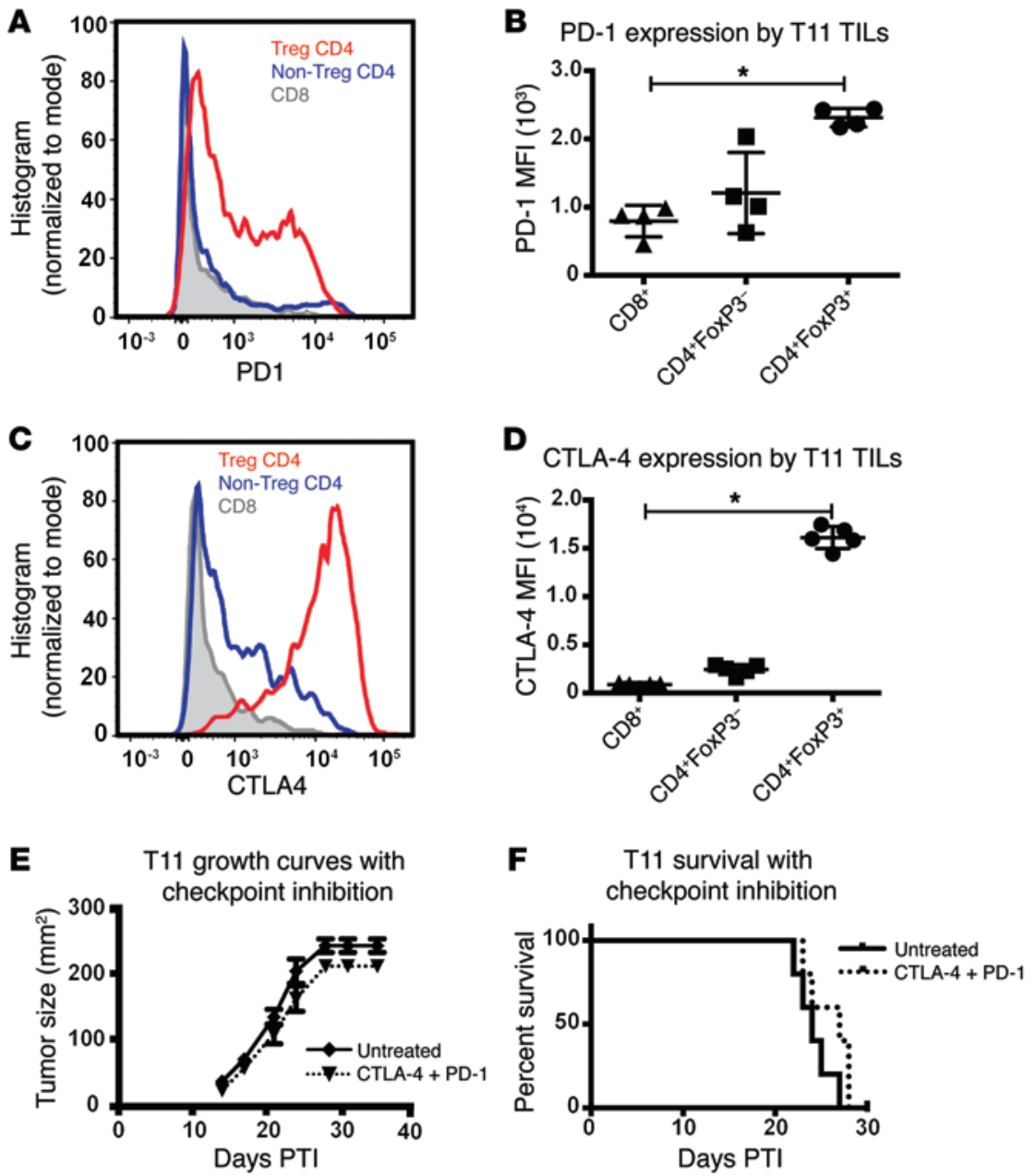

Figure 6. Tumor-infiltrating Tregs express increased levels of PD-1 and CTLA-4; however, PD-1 and CTLA-4 inhibition does not increase survival in T11 tumor-bearing mice. (A) Representative FACS overlay and (B) total MFI values of PD-1 expression by T11 TILs at $20 \mathrm{~mm}^{2} . n=4$. (C) Representative FACS overlay and (D) total MFI values of CTLA- 4 expression by T11 TILs at $20 \mathrm{~mm}^{2} . n=5$. (E) Growth curves of T11 tumor-bearing mice receiving anti-PD-1 and anti-CTLA-4. (F) Survival analysis of data presented in $\mathbf{E}$ ( $n=5$ for each group). Statistical significance was determined by Mann-Whitney $U$ test. Data are presented as mean \pm SEM. ${ }^{*} P<0.05$.

Tregs dominated the immune response, which was associated with diminished numbers of $\mathrm{CD}^{+}{ }^{+} \mathrm{B}$ cells and $\mathrm{CD} 8^{+} \mathrm{T}$ cells. Thus, there may be a critical early period when tumors can recruit sufficient numbers of Tregs to abrogate a functional adaptive immune response.

We sought to determine the mechanism that accounts for the preferential recruitment of Tregs to claudin-low tumors. CXCL12, a chemokine critical for the homing of hematopoietic stem cells (32), was expressed in mice 22-fold more with claudin-low compared with Neu tumors. Further, Tregs that infiltrated claudin-low tumors expressed high levels of CXCR4, one of the receptors for CXCL12. Inhibition of CXCR4 with AMD3100 demonstrated a modest role for this axis in Treg recruitment. AMD3100-treated mice demonstrated a trend for decreased Treg numbers in the tumor

widely by subtype. The use of mouse models that mimic human tumor biology is critical for finding pathways important for tumor pathogenesis and potential. The GEMMs utilized by our laboratory are derived from and studied in immunocompetent mice that recapitulate tumor biology at the genetic and whole tumor level. As expected by similarity to the human patient samples, murine tumors exhibited substantial differences in immune cell infiltration based on intrinsic subtype.

Recently, there has been intense interest in biomarkers of response to checkpoint inhibitor therapy. One stratification that has been proposed for patients with melanoma is immuneexcluded versus immune-infiltrated tumors, with data suggesting that checkpoint inhibition is not effective in immune-excluded tumors (28). Our data would suggest that a third group of tumors, those that are actively immune suppressed, may also not respond to checkpoint inhibition. The presence of Tregs as a biomarker for antitumor response has been confusing, with data indicating both negative and positive clinical benefits (29-31). This may be due to the critical issue of timing in the evaluation of Tregs from the TME. In claudin-low murine tumors, Tregs made up as much as $60 \%$ of all $\mathrm{CD}^{+}$cells in the tumor and were present in substantial numbers prior to the expansion of other adaptive immune cells. These cells were both functional and durable, with continued presence as the tumors grew. In many of the T11 and T12 tumors, compared with controls, which contained elevated PD $-1^{+}$Tregs. However, the modest decrease in the recruitment of Tregs found using AMD3100 was not sufficient to augment the antitumor immune response using checkpoint inhibitor therapy, in part because exposure to AMD3100 also diminished the recruitment of $\mathrm{CD}^{+}$non-Tregs and $\mathrm{CD} 8^{+} \mathrm{T}$ cells to the TME. Similarly, selective inhibition of $\mathrm{p} 110 \delta$ isoform of PI3K using a small molecule inhibitor plus checkpoint inhibition reduced the number of Tregs, which was associated with a significant $(P<0.05)$ improvement in tumor growth compared with that in control mice receiving checkpoint inhibition alone. Unfortunately, this combination did not result in tumor regression or enhanced overall survival. Our data suggest that robust and specific Treg depletion is necessary to engender an effective antitumor response. This level of depletion in our mouse studies was associated with profound autoimmunity. Thus, it is not clear whether prolonged, robust Treg depletion in patients could be tolerated to enhance checkpoint inhibitor therapy for the treatment of claudin-low tumors.

The function of PD-1 on the surface of Tregs is not entirely known $(33,34)$. PD- $1^{\text {hi }}$ Tregs in the TME in glioma patients have been found to be exhausted, yet capable of generating proinflammatory cytokines, such as IFN- $\gamma$ (35). This suggests that one mechanism for the impaired function of anti-PD-1 antibody therapy is the presence of PD-1-expressing Tregs that can (a) bind antibody 
A

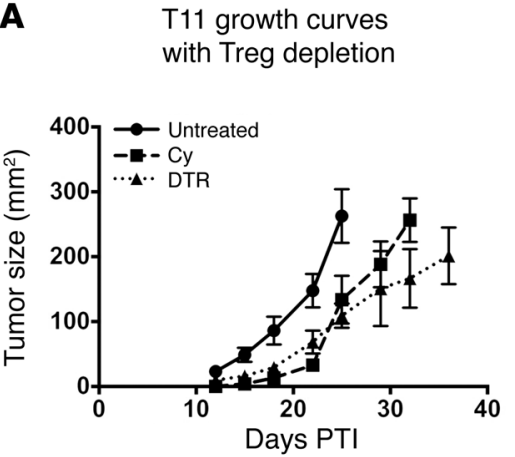

B

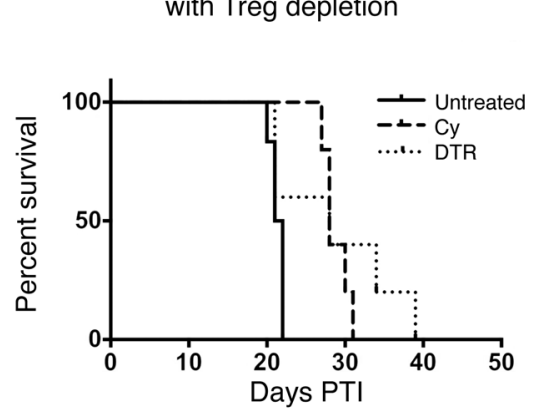

T11 survival with Treg depletion

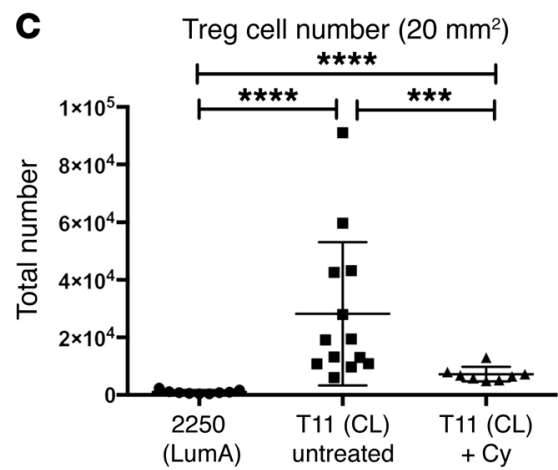

Figure 7. Treg depletion delays tumor growth in the absence of adoptive transfer. WT untreated, Cy-treated, and DEREG mice were injected with $1 \times 10^{4}$ T11 cells ( $n=5$ per group). Cy-treated mice received $100 \mathrm{mg} / \mathrm{kg}$ Cy on day +2 . DEREG mice received $1 \mu \mathrm{g}$ DT on days $-1,0,+6$, and +7 . (A) Growth curves. (B) Survival. There was a significant difference in survival between both Treg-depleted groups compared with the untreated control group $(P<0.05$; log-rank test), but not between treated groups. (C) Treg numbers following a single dose of Cy were reduced compared with those in untreated T11 tumors. Statistical significance was determined by Mann-Whitney $U$ test. Data are presented as mean $\pm \mathrm{SEM}$. ${ }^{* * *} P<0.001 ;{ }^{* * *} P<0.0001$.

if PD-1 is preferentially expressed on these cells and (b) become activated in the presence of anti-PD- 1 antibody. Our data requiring depletion of Tregs to mediate the antitumor activity of antiPD-1 antibody therapy would be consistent with this mechanism.

In summary, we have found that claudin-low tumors are highly enriched with Tregs. Enhancing the immune response to these tumors by depleting Tregs in addition to immune checkpoint inhibition impaired tumor growth and prolonged survival, but was insufficient to mediate tumor regression. Generation of CXCL12 by the tumor cells played a modest role in the recruitment of Tregs to the tumor site. These studies suggest that future clinical trials for patients with triple-negative breast cancer should target the immunosuppressive environment generated by the tumor in combination with checkpoint inhibition.

\section{Methods}

Mice and cell lines. BALB/cJ and C57BL/6J (B6) females and C.B6- $\mathrm{Tg}(-$ Foxp3-DTR/EGFP) 23.2spar/Mmjax (DEREG) females were purchased from The Jackson Laboratory. Neu-N mice were purchased from Charles River Laboratory. Female mice (8 to 14 weeks) were used for all experiments. Rat Neu-expressing NT2 cells (HER2/Neu) and the 2250 (luminal A), 2225 (basal-like), and T11 (claudin-low) tumor models have been described (36-38). T12 cells were prepared by harvesting a T12 tumor from a tumor-bearing mouse, followed by manual digestion with razor blades and chemical digestion with Liberase TM (Roche) and DNase I (Sigma-Aldrich). Digestion was stopped by the addition of EDTA. The 2250 cells were prepared by harvesting a 2250 tumor from a tumor-bearing mouse, followed by manual digestion with razor blades and chemical digestion as described above. BALB/c mice were injected with $1 \times 10^{6} 2250$ cells (luminal A) in Matrigel HC low-growth factor, $1 \times$ $10^{4}$ or $1 \times 10^{5} \mathrm{~T} 12$ (claudin-low) cells in Matrigel HC low-growth factor, or $1 \times 10^{4} \mathrm{~T} 11$ (claudin-low) cells in PBS. Neu-N mice were injected with $5 \times 10^{4} \mathrm{NT} 2$ cells $(\mathrm{Neu})$ in PBS. Tumors were orthotopically transplanted by intradermal injection into a mammary fat pad and measured as previously described (11). Blood and serum samples were harvested by cardiac puncture at the termination of the experiment.

Human breast tumor microarray data sets. All human tumor and normal tissue samples were obtained from fresh-frozen invasive breast carcinomas that were profiled, as described previously, using oligo microarrays (Agilent Technologies) $(18,19)$. We used the microarray data set previously published as the UNC337 data set in the NCBI's Gene Expression Omnibus (GEO GSE18229) (4). All microarray and patient clinical data are available in the University of North Carolina Microarray Database (ref. 20; https://genome.unc.edu/cgi-bin/SMD/ publication/viewPublication.pl?pub_no=81). The probes for all analyses were filtered by requiring the lowest normalized intensity values in both sample and control to be greater than 10. The normalized $\log _{2}$ ratios cyanine-5 (Cy5) dye intensity for experimental sample/ cyanine-3 (Сy3) dye intensity for reference of probes mapping to the same gene (EntrezGene ID as defined by the manufacturer) were averaged to generate independent expression estimates. The METABRIC data set contains 1,981 samples derived from breast tumors of patients participating in the METABRIC trial (39). Gene expression data were acquired by Illumina HT- 12 .

Mouse breast tumor microarray data sets. All mouse samples from fresh-frozen invasive breast carcinomas were analyzed as described previously, using Agilent mouse oligo microarrays (18). Data normalization and preprocessing were identical to those described for the UNC337 data set. We used 2 samples obtained from the combined data set previously published (GEO GSE3165, GSE8516, GSE9343, GSE14457, GSE15263, GSE17916, GSE27101, and GSE42640) (21). The remaining 57 samples represent newly obtained tumor samples using methods approved by IACUC guidelines. Total RNA was purified from 20 to $30 \mathrm{mg}$ of mouse mammary tumor using the QIAGEN RNeasy Mini Kit following the manufacturer's protocols. RNA quantity and quality were determined using the Nanodrop spectrophotometer and Agilent Bioanalyzer, respectively. Total RNA was reverse transcribed and labeled with Cy5 dye for experimental samples and Cy3 dye for mouse reference samples using the Agilent Low RNA Input Fluorescent Linear Amplification Kit (18). Equal quantities of labeled mouse reference RNA and tumor RNA were cohybridized overnight to Agilent microarrays, washed, and scanned; signal intensities were then determined.

Microarray data analysis. Hierarchical clustering of the UNC337 data set to group samples by intrinsic subtype was performed using the intrinsic gene list as described. (22). Centroid linkage hierarchical clustering was performed using Cluster v3.0 (23). Student's $t$ tests for gene expression data were performed using R (24). 
A

T11 survival with Treg depletion and checkpoint inhibition

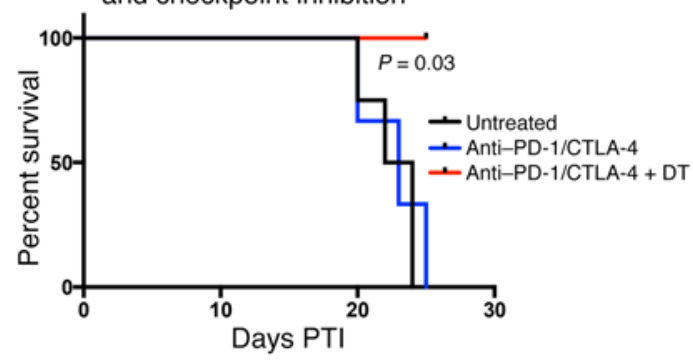

B

B T11 growth curve with Treg depletion and checkpoint inhibition

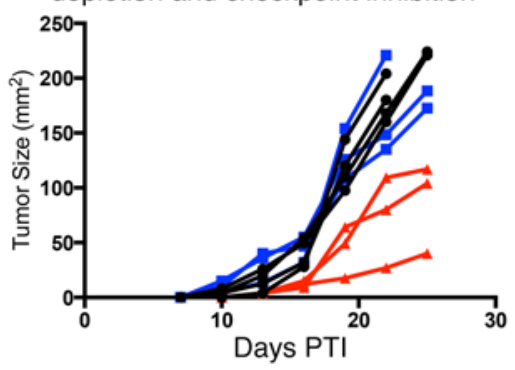

D
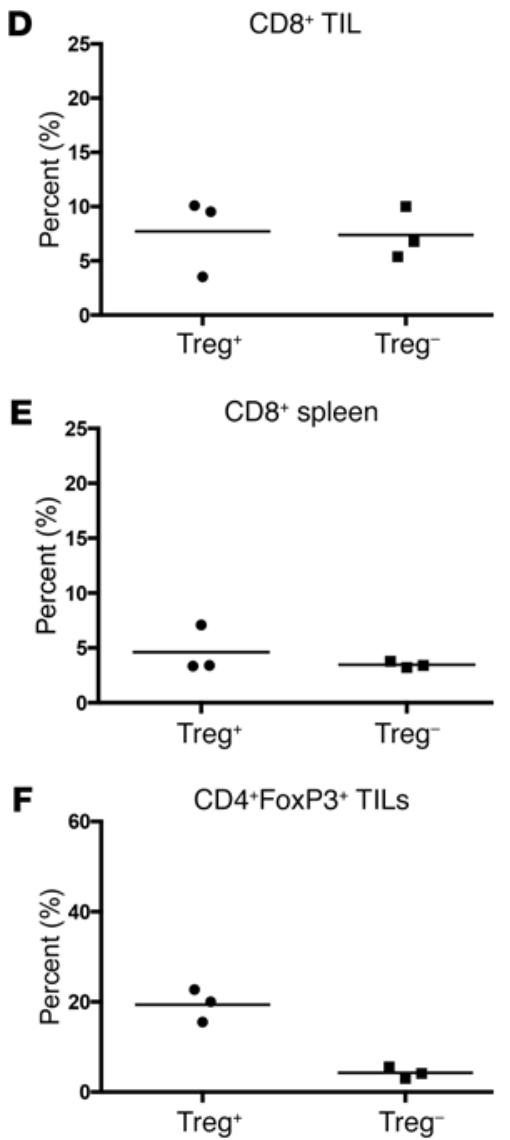

C

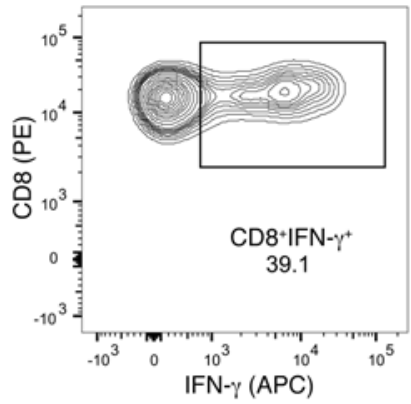

TILs

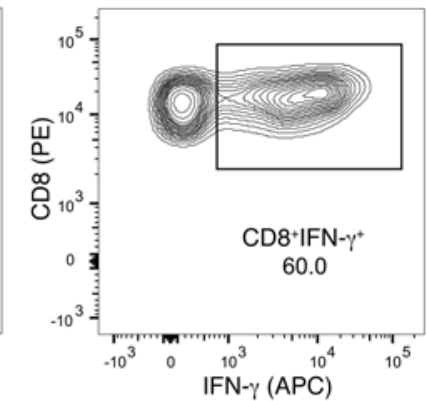

Spleen
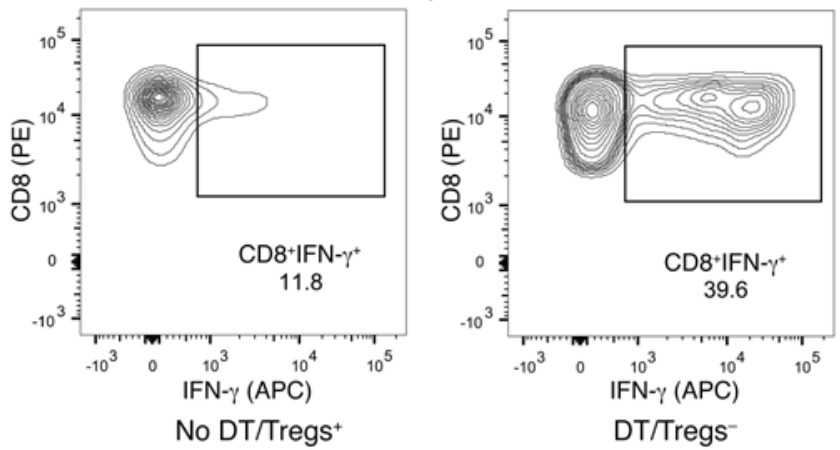
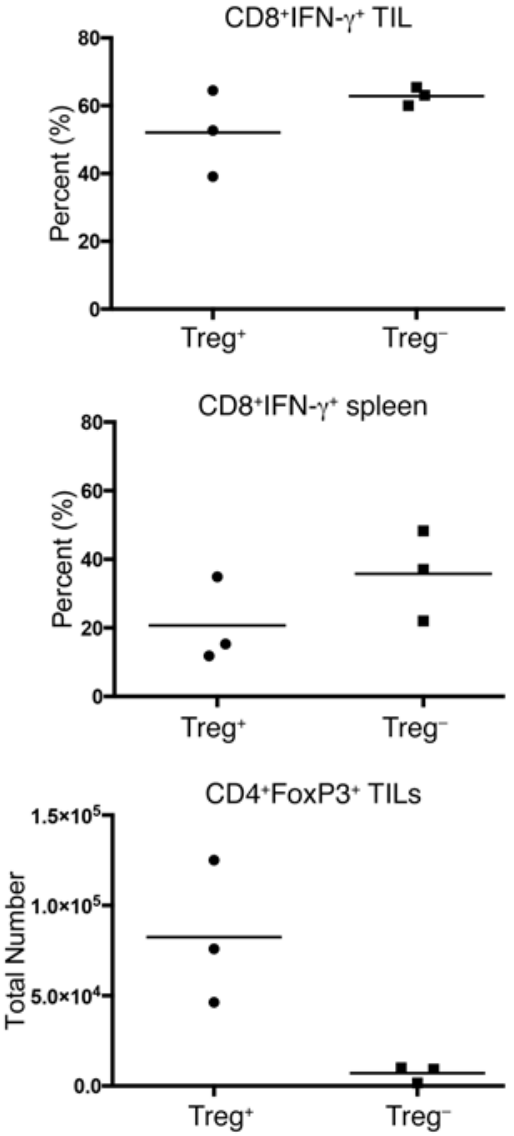

Figure 8. Treg depletion with anti-PD-1 and anti-CTLA-4 immune checkpoint inhibition significantly delays T11 tumor growth. WT or FoxP3-DTR mice were injected with $1 \times 10^{4}$ T11 cells. DEREG mice received $1 \mu \mathrm{g}$ DT on days -1 , 0,6 , and 7 PTI. DT + PD- $1+$ CTLA-4 mice received $1 \mu \mathrm{g}$ DT on days $-1,0,6$, and 7 PTI and anti-PD-1 and antiCTLA- 4 antibody on day -1 , then every other day for the duration of the experiment. Tumor growth evaluation was terminated at day 25 PTI due to autoimmune manifestations and tumor growth reaching an end point of 170 $\mathrm{mm}^{2}$. (A) Mice depleted of Tregs and receiving anti-PD-1 and anti-CTLA-4 $(n=3)$ had a significant survival benefit compared with untreated mice $(n=4)$ or those treated with anti-PD- 1 and anti-CTLA-4 alone $(n=3)$ (CLTLA4 + PD-1 vs. DT + CLTLA4 + PD-1: $P=0.03$; log-rank test). (B) Individual replicates of tumor growth curves. (C-E) Mice treated with anti-PD-1 and anti-CTLA-4 alone (Treg) ( $n$ = 3) or anti-PD-1 and anti-CTLA-4 with DT (Treg-) $(n=3)$. Mice were sacrificed 25 days PTI and FACS analyzed for percentage of $\mathrm{CD} 8^{+} \mathrm{T}$ cells and IFN- $\gamma$ production by $\mathrm{CD} 8^{+}$ T cells in tumor and spleen. (C) Representative FACS plot of IFN- $\gamma$ production by $\mathrm{CD} 8^{+} \mathrm{T}$ cells from tumor or spleen of tumor-bearing mice. (D) Percentage of $\mathrm{CD}^{+} \mathrm{T}$ cells and IFN- $\gamma$ production by CD8 ${ }^{+} T$ cells from tumor. (E) Percentage $\mathrm{CD} 8^{+} \mathrm{T}$ cells and IFN- $\gamma$ production by $\mathrm{CD} 8^{+} T$ cells in spleen. (F) Percentage and total number of CD4 ${ }^{+} \mathrm{FoxP} 3$ Tregs from tumors in mice treated with anti-PD-1 and anti-CTLA-4 alone (Treg') $(n=3)$ or anti-PD-1 and antiCTLA-4 with DT (Treg-) $(n=3)$. Analysis of T cell TILs was not possible after day 25 in DT-treated mice due to severe autoimmunity necessitating termination of the experiment at that time. Data are presented as mean \pm SEM.

$H \& E$ and IF analyses of patient samples. Staining was performed on tumor-infiltrating lymphocytes in 85 breast cancer whole tissue sections (WTS) obtained from UNC hospitals surgical pathology archive. IHC and dual IF on WTS with immune infiltrates were performed with the Bond fully automated slide-staining system using the Bond Polymer Refine Detection Kit (DS9800, Leica Microsystems). Slides were deparaffinized in Bond Dewax Solution (AR9222) and hydrated in Bond Wash Solution (AR9590). Antigen retrieval was done at $100^{\circ} \mathrm{C}$ either in Bond Epitope Retrieval Solution 1, $\mathrm{pH}$ 6.0 (AR9661), or in Solution 2, pH 9.0 (AR9640). IHC slides were 
visualized with DAB and counterstained with hematoxylin using Bond Polymer Refine Detection. Stained slides were dehydrated. IF slides were counterstained with Hoechst 33258 (Invitrogen) and mounted with ProLong Gold antifade reagent (P36934, Life Technologies). Tumor tissue slides ( $n=10$ per subtype) were stained for CD8, CD4, and FoxP3 and analyzed for coexpression at the cellular level using TissueStudio on-slide cytometry to measure tumor infiltration by Tc cells $\left(\mathrm{CD}^{+}\right)$, Th cells $\left(\mathrm{CD}^{+}{ }^{+} \mathrm{FoxP} 3^{-}\right)$, and Tregs $\left(\mathrm{CD} 4^{+} \mathrm{FoxP}^{+}\right)$. All samples were evaluated by pathologists blinded to the tumor subtype.

Imaging and digital image analysis. $\mathrm{H} \& \mathrm{E}$ and $\mathrm{IF}$-stained slides (CD4, CD8, CD19, and FoxP3) were digitally imaged at 20× magnification using the Aperio ScanScopes XT and FL (Aperio Technologies). The hematoxylin and DAB OD parameters were adjusted for both algorithms. The nuclear segmentation factor and the weak positive threshold were tuned for the cytoplasmic v2 and the color deconvolution v9 algorithms, respectively. High-resolution acquisition (20x objective) of the stained slides in the DAPI, Cy3, and Cy5 channels was performed in the Aperio ScanScope FL (Aperio Technologies). Cell nuclei were visualized in the DAPI channel; CD8, CD19, and FoxP3 were visualized in the Cy3 channel; CD4 was visualized in the Cy5 channel. To determine the number of cells coexpressing FoxP3/ CD4, slides were analyzed using Definiens Tissue Studio image analysis software (Architect XD v 2.0.4, Tissue Studio v 3.5).

Antibodies and flow cytometry reagents: human antibodies. Mouse monoclonal antibodies against human CD4 (clone 4B12), CD8 (clone 4B11), and CD19 (clone BT51E) were purchased from Leica Microsystems. FoxP3 (clone 236A/E7) was purchased from Abcam. CD279 (PD-1, clone J105) was purchased from eBioscience.

Antibodies and flow cytometry reagents: mouse antibodies. Flow cytometry monoclonal antibodies against murine CD4 (GK1.5 or RM4-5), CD8 (53-6.7), PD-1 (J43 or RMP1-30), CD45 (30-F11), CD62L (MEL-14), and CXCR4 (2B11) were purchased from eBioscience. CD19 (6D5) and CD44 (IM7) were purchased from BioLegend. Intracellular antibodies FoxP3 (FJK-16s), TNF (MP6-XT22), IFN- $\gamma$ (XMG1.2), and Helios (22F6) were purchased from eBioscience. Cell viability was determined using Blue or Aqua Fluorescence Reactive Dye (Life Technologies).

Flow cytometry. Cells were surface stained, fixed, and permeabilized overnight using the Foxp3/Transcription Factor Staining Buffer Set (eBioscience), and intracellular staining was performed the following day according to the manufacturer's instructions. Data were acquired using MACSQuant (Miltenyi Biotec), BD Canto, or LSR II (BD Biosciences). Acquired data were analyzed using FlowJo Flow Cytometry Analysis Software (Tree Star Inc.).

Isolation of murine TILs. Murine tumors were resected and digested in Liberase TM (Roche), DNase I (Sigma-Aldrich), hyaluronidase (Sigma-Aldrich), and collagenase XI (Sigma-Aldrich), as previously described (25). Single-cell suspensions were enriched for lymphocytes by isolating cells at the interface of $44 \%$ Percoll (SigmaAldrich) in media and Lympholyte-M (Cedarlane) gradient. Isolated cells were stimulated for 4 hours in PMA/ionomycin prior to intracellular cytokine staining.

In vivo cell inhibition and depletion. Monoclonal antibodies used for in vivo antibody inhibition and depletion were purchased from BioXCell or obtained from the laboratory of Bruce Blazar (University of Minnesota, Minneapolis, Minnesota, USA) (anti-PD-1 mAb). Mice undergoing immune checkpoint inhibition received i.p. injection of $100 \mu \mathrm{g}$ antiCTLA-4 (9D9), $200 \mu \mathrm{g}$ anti-PD-1 (J43), or isotype antibody on day -1 PTI and then every other day throughout the experiment $(40,41)$.

Mice undergoing Treg depletion (DEREG mice) received i.p. injections of $1 \mu \mathrm{g}$ DT in PBS on days $-1,+1,+6$, and +7 (27). Mice undergoing specific inhibition of p110 $\delta$ PI3K were given drug $(75 \mathrm{mg} / \mathrm{kg}$ PI-3065, once daily) or vehicle ( $0.5 \%$ methylcellulose with $0.2 \%$ Tween 80 ) by oral gavage from day +1 through the duration of the experiment (26).

Treg-suppression assay. For the Treg-suppression assays, we evaluated splenic Tregs. FoxP ${ }^{+} \mathrm{GFP}^{+}$cells were sorted using a MoFlo Cell Sorter (Beckman Coulter). Stimulator cells were isolated from WT B6 splenocytes following CD90 microbead depletion (Miltenyi Biotec) and irradiation at 2,100 centigray (cGy). Responder cells were isolated from WT BALB/c mice using the Cedarlane T Recovery Column Kit. Isolated cells were then B220 and CD25 depleted using phycoerythrin-conjugated (PE-conjugated) antibodies and antiPE magnetic bead sorting (Miltenyi Biotec). Responder cells were stained with the Cell Proliferation Dye eFluor 670 (eBioscience) and plated at varying Treg/Teff ratios. Cells were cocultured for 3 days, stained, and FACS analyzed.

Real-time PCR array. Whole tumor RNA was isolated using the RNEasy Kit (QIAGEN) and reverse transcribed. Real-time PCR was performed on the ABI 7300 (Applied Biosystems Inc.), using Master Mix from the Mouse Inflammatory Cytokines and Receptors (PAMM-011) RT2 Profiler PCR Array System (QIAGEN). Ct values were determined by ABI software. Data analysis was performed using the web-based RT2 Profiler PCR Array Data Analysis version 3.5 (QIAGEN).

AMD3100 and Cy. Alzet osmotic pumps, model 2002 (Alza), were loaded with $10 \mathrm{mg}$ AMD3100 (Tocris) dissolved in PBS. Control mice received pumps loaded with PBS without AMD3100 (28). Pumps were subcutaneously implanted dorsally on day -2 . Mice were then challenged with $1 \times 10^{4} \mathrm{~T} 11$ cells, and tumors were harvested 12 days later at approximately $20 \mathrm{~mm}^{2}$ in accordance with IACUC guidelines. For low-dose Cy studies, a single dose of $100 \mathrm{mg} / \mathrm{kg}$ Cy was given PTI. This dose was sufficient to deplete $60 \%$ of the Tregs for approximately 8 days with rebound increases above baseline at day 12 after therapy (data not shown).

CXCL12 knockdown. T11 cells were treated with $4 \mu \mathrm{g} / \mathrm{ml}$ Polybrene and cultured overnight with Mission shRNA Lentiviral Transduction Particles (Sigma-Aldrich) containing shRNA specific for CXCL12 or a nontarget control (scramble) at an MOI of 10. Lentiviral particles were removed the next morning and new medium was added. The following day, cells were cultured in medium containing $6 \mu \mathrm{g} / \mathrm{ml}$ puromycin and expanded to select for transduced cells. Cells were tested for CXCL12 production by real-time PCR and ELISA. Two different knockdown cell lines with different levels of knockdown of CXCL12, as evaluated by ELISA assay, were characterized. Knockdown of CXCL12 had no impact on the growth of T11 cells in vitro (data not shown).

Statistics. Data are presented as mean \pm SEM. Statistical differences were determined using Student's $t$ test, Mann-Whitney $U$ test, or Kruskal-Wallis test, with Dunn's post-test for multiple comparisons where appropriate. Survival data are presented using Kaplan-Meier survival analysis. Survival results were analyzed using the log-rank test. Statistical analyses were conducted using GraphPad Prism 6 software, unless otherwise noted. Results were considered statistically significant at $P \leq 0.05$. 
Study approval. All animal experiments were conducted in accordance with protocols approved by the UNC IACUC. All human tumor and normal tissue samples were collected using IRB-approved protocols.

\section{Author contributions}

NAT and SCV designed and performed experiments, analyzed data, and wrote the manuscript. MDI and BRM performed experiments and analyzed data. WJB, KPM, SR, and JSP analyzed data. CKA and LAC provided clinical samples for analysis. BGV designed experiments, analyzed data, and wrote and edited the manuscript. CMP and JSS conceived of the project, analyzed data, and edited the manuscript.

\section{Acknowledgments}

The UNC Flow Cytometry Core Facility is supported in part by a P30 CA016086 Cancer Center Core Support grant to the UNC Lineberger Comprehensive Cancer Center. This work is supported by Breast Cancer SPORE (P50CA058223 CMP to JSS), the UNC Oncology Clinical Translational Research Training Program (5K12CA120780 to BGV), and the University Cancer Research Fund (to JSS and BGV).

Address correspondence to: Jonathan Serody, Lineberger Comprehensive Cancer Center, 450 West Drive, CB7295, University of North Carolina, Chapel Hill, North Carolina 27599, USA. Phone: 919.445.4175; Email: jonathan_serody@med.unc.edu.
1. Cancer Facts \& Figures. American Cancer Society. https://www.cancer.org/content/dam/cancer-org/ research/cancer-facts-and-statistics/annual-cancer-facts-and-figures/2016/cancer-facts-andfigures-2016.pdf. Accessed August 1, 2017.

2. Bianchini G, Balko JM, Mayer IA, Sanders ME, Gianni L. Triple-negative breast cancer: challenges and opportunities of a heterogeneous disease. Nat Rev Clin Oncol. 2016;13(11):674-690.

3. Nottegar A, et al. Extra-nodal extension of sentinel lymph node metastasis is a marker of poor prognosis in breast cancer patients: A systematic review and an exploratory meta-analysis. Eur J Surg Oncol. 2016;42(7):919-925.

4. Kos Z, Dabbs DJ. Biomarker assessment and molecular testing for prognostication in breast cancer. Histopathology. 2016;68(1):70-85

5 . Savas P, et al. Clinical relevance of host immunity in breast cancer: from TILs to the clinic. Nat Rev Clin Oncol. 2016;13(4):228-241.

6. Campbell JB. Breast cancer-race, ethnicity, and survival: a literature review. Breast Cancer Res Treat. 2002;74(2):187-192.

7. Baum $\mathrm{M}$. The changing face of breast cancer - past, present and future perspectives. Breast Cancer Res Treat. 2002;75(Suppl 1):S1-S5; discussion S33.

8. Anders CK, Zagar TM, Carey LA. The management of early-stage and metastatic triple-negative breast cancer: a review. Hematol Oncol Clin North Am. 2013;27(4):737-749, viii.

9. Prat A, Adamo B, Cheang MC, Anders CK, Carey LA, Perou CM. Molecular characterization of basal-like and non-basal-like triple-negative breast cancer. Oncologist. 2013;18(2):123-133.

10. Carey L. Old drugs, new tricks for triple-negative breast cancer. Lancet Oncol. 2015;16(4):357-359.

11. Newman LA, Reis-Filho JS, Morrow M, Carey LA, King TA. The 2014 Society of Surgical Oncology Susan G. Komen for the Cure Symposium: triple-negative breast cancer. Ann Surg Oncol. 2015;22(3):874-882

12. Wallden B, et al. Development and verification of the PAM50-based Prosigna breast cancer gene signature assay. BMC Med Genomics. 2015;8:54.

13. Prat A, Ellis MJ, Perou CM. Practical implications of gene-expression-based assays for breast oncologists. Nat Rev Clin Oncol. 2011;9(1):48-57.

14. Iglesia MD, Parker JS, Hoadley KA, Serody JS, Perou CM, Vincent BG. Genomic analysis of immune cell infiltrates across 11 tumor types. J Natl Cancer Inst. 2016;108(11):djw144.
15. Iglesia MD, et al. Prognostic B-cell signatures using mRNA-seq in patients with subtype-specific breast and ovarian cancer. Clin Cancer Res. 2014;20(14):3818-3829.

16. Prat A, et al. Phenotypic and molecular characterization of the claudin-low intrinsic subtype of breast cancer. Breast Cancer Res. 2010;12(5):R68.

17. Purrington KS, et al. Genes associated with histopathologic features of triple negative breast tumors predict molecular subtypes. Breast Cancer Res Treat. 2016;157(1):117-131.

18. Stanton SE, Adams S, Disis ML. Variation in the incidence and magnitude of tumor-infiltrating lymphocytes in breast cancer subtypes: a systematic review. JAMA Oncol. 2016;2(10):1354-1360.

19. Rody A, et al. A clinically relevant gene signature in triple negative and basal-like breast cancer. Breast Cancer Res. 2011;13(5):R97.

20. Gatza ML, Silva GO, Parker JS, Fan C, Perou CM. An integrated genomics approach identifies drivers of proliferation in luminalsubtype human breast cancer. Nat Genet. 2014;46(10):1051-1059.

21. Sørlie T, et al. Gene expression patterns of breas carcinomas distinguish tumor subclasses with clinical implications. Proc Natl Acad Sci U S A. 2001;98(19):10869-10874.

22. Perou CM, et al. Molecular portraits of human breast tumours. Nature. 2000;406(6797):747-752

23. Hu Z, et al. The molecular portraits of breast tumors are conserved across microarray platforms. BMC Genomics. 2006;7:96.

24. Herschkowitz JI, et al. Comparative oncogenomics identifies breast tumors enriched in functional tumor-initiating cells. Proc Natl Acad Sci US A. 2012;109(8):2778-2783.

25. Burgents JE, Moran TP, West ML, Davis NL, Johnston RE, Serody JS. The immunosuppressive tumor environment is the major impediment to successful therapeutic vaccination in Neu transgenic mice. J Immunother. 2010;33(5):482-491.

26. Ali K, et al. Inactivation of $\mathrm{PI}(3) \mathrm{K}$ p $110 \delta$ breaks regulatory T-cell-mediated immune tolerance to cancer. Nature. 2014;510(7505):407-411.

27. Sharma P. Biology and management of patients with triple-negative breast cancer. Oncologist. 2016;21(9):1050-1062.

28. Spranger S, Gajewski TF. Tumor-intrinsic oncogene pathways mediating immune avoidance. Oncoimmunology. 2016;5(3):e1086862.

29. Liu S, et al. Prognostic significance of FOXP3+ tumor-infiltrating lymphocytes in breast cancer depends on estrogen receptor and human epidermal growth factor receptor-2 expression status and concurrent cytotoxic T-cell infiltration. Breast Cancer Res. 2014;16(5):432.

30. Andre F, et al. Molecular pathways: involvement of immune pathways in the therapeutic response and outcome in breast cancer. Clin Cancer Res. 2013;19(1):28-33.

31. West NR, et al. Tumour-infiltrating FOXP3(+) lymphocytes are associated with cytotoxic immune responses and good clinical outcome in oestrogen receptor-negative breast cancer. $\mathrm{Br} \mathrm{J}$ Cancer. 2013;108(1):155-162.

32. Ugarte F, Forsberg EC. Haematopoietic stem cell niches: new insights inspire new questions. EMBO J. 2013;32(19):2535-2547.

33. Park HJ, et al. PD-1 upregulated on regulatory $\mathrm{T}$ cells during chronic virus infection enhances the suppression of CD8+ $\mathrm{T}$ cell immune response via the interaction with PD-L1 expressed on CD8+ T cells. JImmunol. 2015;194(12):5801-5811.

34. Zhou Q, et al. Program death-1 signaling and regulatory $\mathrm{T}$ cells collaborate to resist the function of adoptively transferred cytotoxic T lymphocytes in advanced acute myeloid leukemia. Blood. 2010;116(14):2484-2493.

35. Lowther DE, et al. PD-1 marks dysfunctional regulatory T cells in malignant gliomas. JCI Insight . 2016;1(5):e85935.

36. Roberts PJ, et al. Combined PI3K/mTOR and MEK inhibition provides broad antitumor activity in faithful murine cancer models. Clin Cancer Res. 2012;18(19):5290-5303.

37. Usary J, Darr DB, Pfefferle AD, Perou CM. Overview of genetically engineered mouse models of distinct breast cancer subtypes. Curr Protoc Pharmacol. 2016;72:14.38.1-14.3811.

38. Moran TP, et al. Alphaviral vector-transduced dendritic cells are successful therapeutic vaccines against neu-overexpressing tumors in wildtype mice. Vaccine. 2007;25(36):6604-6612.

39. Curtis C, et al. The genomic and transcriptomic architecture of 2,000 breast tumours reveals novel subgroups. Nature. 2012;486(7403):346-352.

40. Dai M, Yip YY, Hellstrom I, Hellstrom KE. Curing mice with large tumors by locally delivering combinations of immunomodulatory antibodies. Clin Cancer Res. 2015;21(5):1127-1138.

41. Li C, et al. ADAP and SKAP55 deficiency suppresses PD-1 expression in CD8+ cytotoxic T lymphocytes for enhanced anti-tumor immunotherapy. EMBO Mol Med. 2015;7(6):754-769. 